\title{
Metric observations of transient, quasi-periodic radio emission from the solar corona in association with a "halo" CME and an "EIT wave" event
}

\author{
R. Ramesh ${ }^{1,2}$, C. Kathiravan ${ }^{2}$, A. Satya Narayanan ${ }^{2}$, and E. Ebenezer ${ }^{2}$ \\ ${ }^{1}$ Centre for Research and Education in Science and Technology, Hosakote 562 114, India \\ 2 Indian Institute of Astrophysics, Bangalore 560 034, India
}

Received 6 May 2002 / Accepted 7 January 2003

\begin{abstract}
We present metric radio observations of quasi-periodic emission from the solar corona in close association with a "halo" CME and an "EIT wave" phenomenon. The radio event lasted for about $60 \mathrm{~s}$. Its mean periodicity was $\approx 8.8 \mathrm{~s}$. The estimated Alfvén speed in the corona was $1034 \mathrm{~km} \mathrm{~s}^{-1}$. The derived height of the source region of the observed quasi-periodic emission, and the magnetic field at the corresponding location (for emission in the fundamental mode) were $\sim 0.23 R_{\odot}$ and $6.3 \mathrm{G}$, respectively. The magnetic field for harmonic emission was found to be $\sim 3.1 \mathrm{G}$.
\end{abstract}

Key words. sun: activity - sun: corona - sun: oscillations - plasmas - magnetohydrodynamics (MHD) - sun: radio radiation

\section{Introduction}

Since the launch of the Solar and Heliospheric Observatory (SOHO) in December 1995, the Extreme-Ultraviolet Imaging Telescope (EIT, Delaboudinière et al. 1995) has recorded numerous instances of wavelike disturbances during the onset of coronal mass ejections (CMEs) observed with the Large Angle and Spectrometric Coronagraph (LASCO, Brueckner et al. 1995). The "EIT waves" (as they are commonly referred to) move away from or near flaring active regions with a typical speed of $\sim 200-500 \mathrm{~km} \mathrm{~s}^{-1}$ (Thompson et al. 1999). It is possible that these values are only a lower limit since the time cadence of the EIT instrument ( 10-15 min per image) makes it difficult to determine precisely the exact start time or origin of the wavelike transients and obtain a reliable estimate of their speed (Biesecker et al. 2002). Recent observations of propagating disturbances in the solar corona with instruments of better time resolution also seems to indicate this (Aschwanden et al. 1999; Wills-Davey \& Thompson 1999; Khan \& Hudson 2000; Pohjolainen et al. 2001; Khan \& Aurass 2002). Again, a statistical study of the "EIT waves" and the associated metric type II radio bursts show that the latter propagates 2-3 times faster (mean speed $\approx 739 \mathrm{~km} \mathrm{~s}^{-1}$ ) than the former (Klassen et al. 2000). Of course, waves are interesting in their own right, simply because they are there, but their use in enlightening of coronal conditions adds greatly to that interest. It is well known that the fundamental plasma parameters in the solar corona are

Send offprint requests to: $\mathrm{R}$. Ramesh, e-mail: ramesh@iiap.ernet.in relevant to the understanding of solar flares, to the acceleration of solar wind and coronal mass ejection (CME), and, ultimately, to Sun-Earth connection. The recent observations of oscillations in coronal loops (Aschwanden et al. 1999) has now raised the feasibility of the development of coronal seismology (see Roberts 2000 for a review on the topic), analogus to helioseismology which provides us with powerful results concerning the interior of the Sun. The seismic information offers the means of determining local conditions, particularly the coronal magnetic field which is generally inferred mainly from measurements of the line-of-sight component of the photospheric magnetic field. The observations of transient, pulsating radio emission from the solar corona is a potential diagnostic tool in this connection since radio data can be obtained with a high temporal and spectral resolution (Roberts et al. 1983). According to Aschwanden (1987), the favourable conditions for MHD oscillations occur mainly in the upper part of the corona (metric range). In this situation, we present here metric radio observations of quasi-periodic radio emission from the solar corona in close temporal and spatial association with a "halo" CME and an "EIT wave" event, and derive the coronal plasma parameters (notably the Alfvén speed and through this the magnetic field strength), using it.

\section{Observations}

The radio data reported were obtained with the Gauribidanur radioheliograph (GRH) operating near Bangalore in India (see Ramesh et al. 1998 for details on the instrument) on 


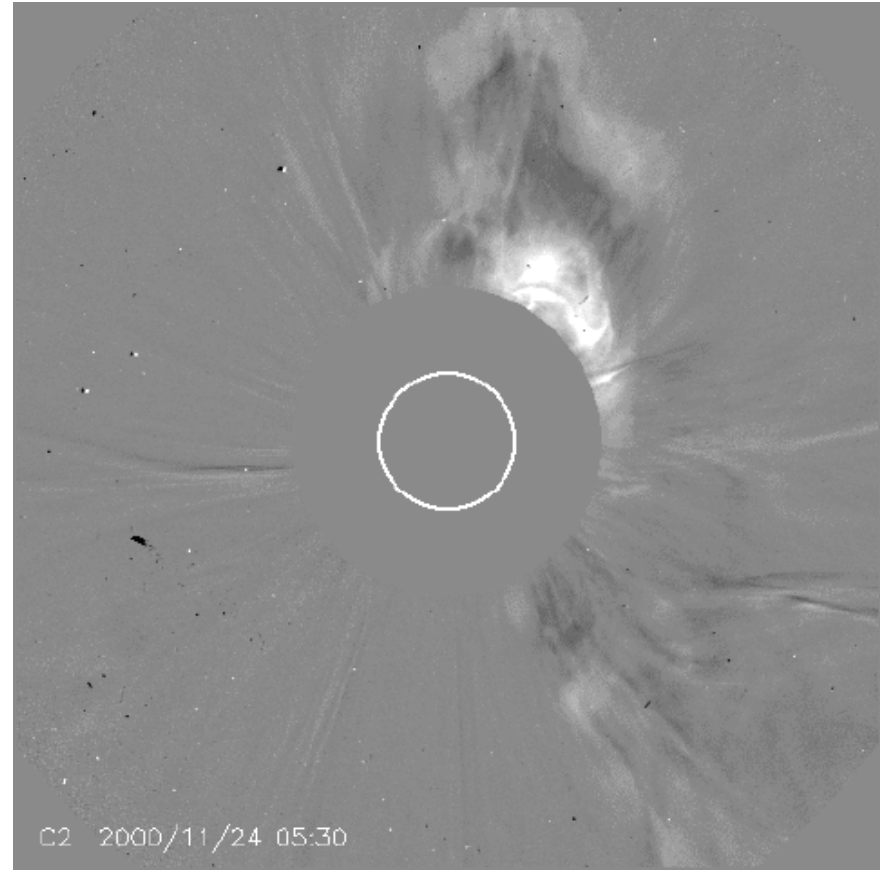

Fig. 1. Difference image (05:30-05:06 UT) of the "halo" CME observed on November 24, 2000 with the LASCO C2 coronagraph onboard SOHO. The inner circle indicates the solar disk, and the outer circle is the occulter of the coronagraph. It extends approximately upto $2.2 R_{\odot}$ from the center of the Sun. Solar north is straight up and east is to the left.

November 24, 2000. The observing frequency was $109 \mathrm{MHz}$. The time resolution and the bandwidth used were $256 \mathrm{msec}$ and $1 \mathrm{MHz}$, respectively. The minimum detectable flux limit of the array is $\sim 0.02 \mathrm{SFU}$, and the angular resolution is $\sim 7^{\prime} \times 11^{\prime}(\mathrm{RA} \times \mathrm{Dec})$ at the above frequency. According to the SOHO/LASCO catalog for the year 2000, a full "halo" CME was observed on November 24, 2000. The event was first observed in the LASCO C2 coronagraph at 05:30 UT over the NW limb (Fig. 1). Its estimated linear speed in the plane of the sky was $994 \mathrm{~km} \mathrm{~s}^{-1}$. The lift-off time of the CME, calculated from an extrapolation of its height-time history was 04:55:52 UT (Fig. 2). A 3B class H $\alpha$ flare (04:57-05:35 UT with peak at 05:01 UT) from AR 9236 located at N20W05, and a GOES X2.0 class X-ray flare (04:55-05:08 UT with peak at 05:02 UT) were reported in association with the above CME event (Solar Geophysical Data, May 2001). It was also accompanied by an "EIT wave" from AR 9236 (http://umbra.nascom.nasa.gov/eit/plan/2000/-

200011/log_form20001127.html) and a coronal type II radio burst (05:02-05:11 UT) in the frequency range 180$30 \mathrm{MHz}$, with an estimated shock speed of $1100 \mathrm{~km} \mathrm{~s}^{-1}$ (Solar Geophysical Data, January 2001). There was also an interplanetary type II burst starting from about 05:10 UT in the range $14 \mathrm{MHz}-100 \mathrm{KHz}$, following the coronal type II event (http://lep694.gsfc.nasa.gov/waves/waves.html).

Figures 3 and 4 show two EIT $195 \AA$ running difference images (04:59-04:47 UT) and (05:11-04:59 UT). The regions marked by arrow mark in Fig. 4 indicate the probable locations of an

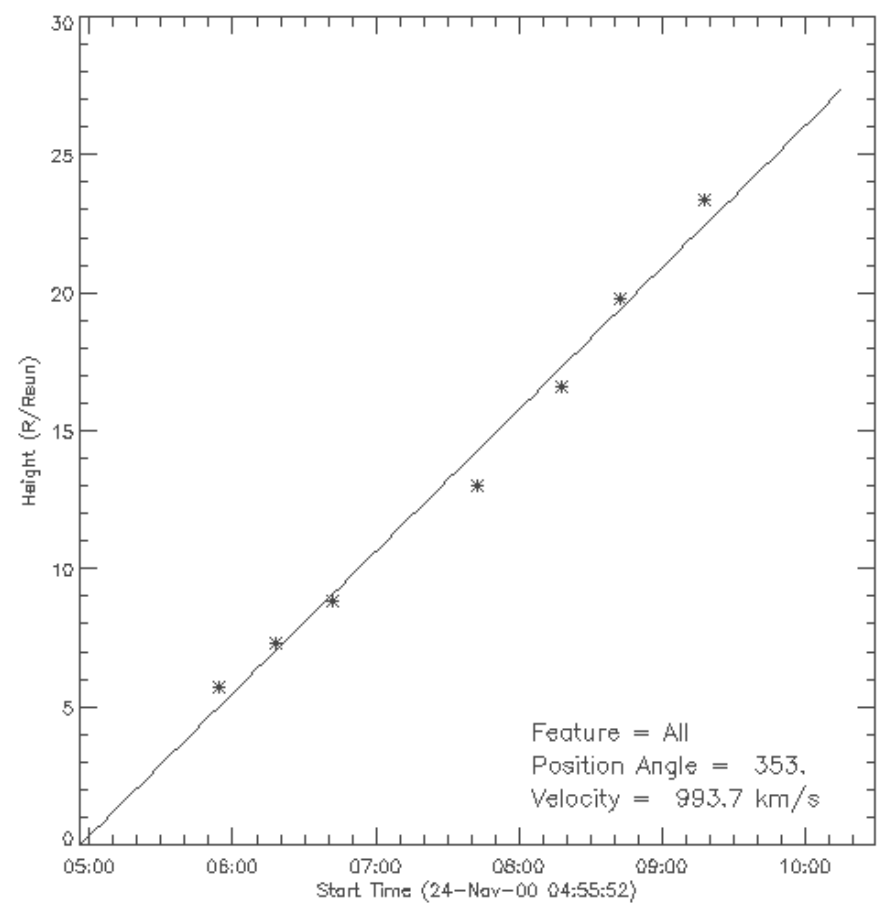

Fig. 2. Height-time plot of the "halo" CME shown in Fig. 1. The asterik marks indicate the location of the leading edge of the CME in the field of view of LASCO C 2 \& C3 coronagraphs. The straight line is a linear fit to the data.

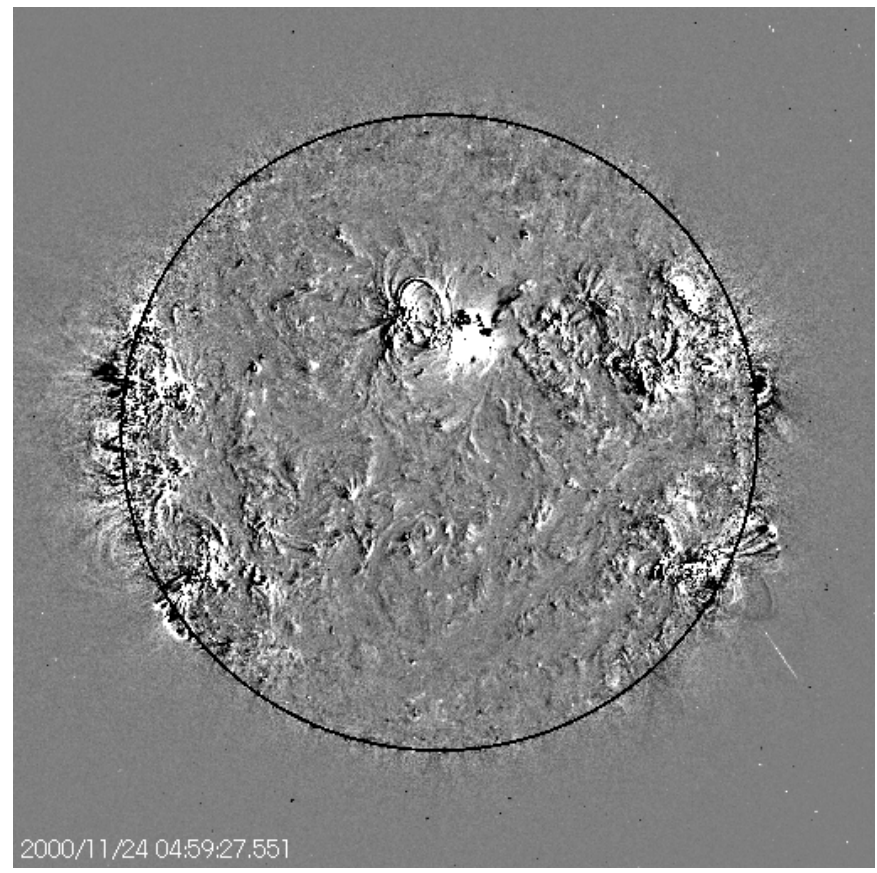

Fig. 3. SOHO-EIT $195 \AA$ running difference image obtained on November 24, 2000 at 04:59 UT by subtracting the 04:47 UT image.

"EIT wave". A comparison of the two figures suggest that the latter should have originated on or before 04:59 UT. Figure 5 shows half-power contours of the radioheliogram obtained with the GRH at 04:58:54, 04:59:21 and 04:59:47 UT. There is a close correspondence between the location of the discrete 


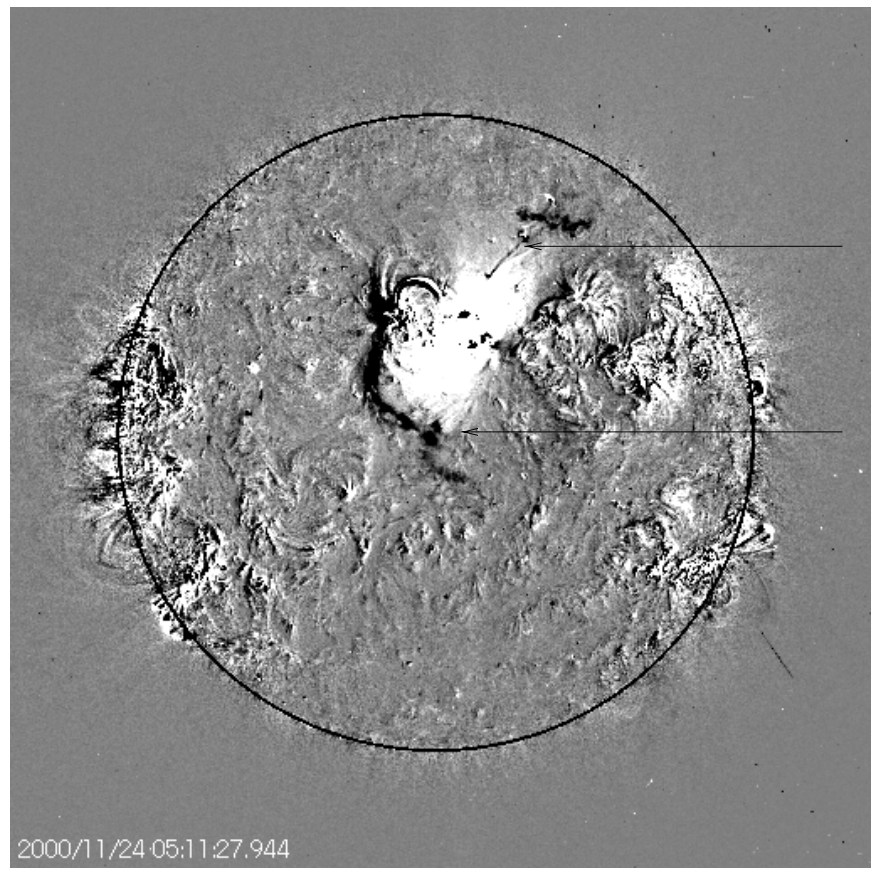

Fig. 4. Same as in Fig. 3, but obtained at 05:11 UT. The regions marked by arrow marks indicate the probable locations of an "EIT wave".

regions of intense emission in the radio image and the flare site mentioned above. The temporal evolution of the whole Sun flux observed with the GRH during the interval 04:5805:01 UT revealed well-defined quasi-periodic emission with a mean period of $\approx 8.8 \mathrm{~s}$. The latter started around 04:58:30 UT and lasted upto 04:59:30 UT (Fig. 6). The observed peak flux density is $\approx 63$ SFU. An inspection of the spectral data obtained with the Hiraiso radiospectrograph (HiRAS) operated by the Hiraiso Solar Terrestrial Reserach Center (Kondo et al. 1995) revealed the presence of a group of fast drifting radio bursts (Fig. 7) around the same time as our observations in Fig. 6. The nature of the emission and its close temporal association with the flare events suggests that it is a type III group (Mangeney \& Pick 1989; Benz 1994). We would like to point here that the Solar Geophysical Data (January 2001) had also reported a type III radio burst group around the same time. It is clear from the above that the radio light curve in Fig. 6 corresponds to the aforementioned burst. The scatter in the centroid of the radio sources in Fig. 5 also points to an association with a type III group, since the latter are considered to escape along large scale diverging magnetic field lines connected to a common acceleration site (Mercier 1975; Roelof \& Pick 1979; Pick \& Ji 1986).

\section{Analysis and results}

It is now well known that metric radio observations of solar type III burst group sometimes show quasi-periodic fluctuations (Wild et al. 1963; Janssens et al. 1973; Mangeney $\&$ Pick 1989). They consist of several widely separated elementary components which radiate quasi-simultaneously or successively (Vlahos et al. 1986 and the references therein).

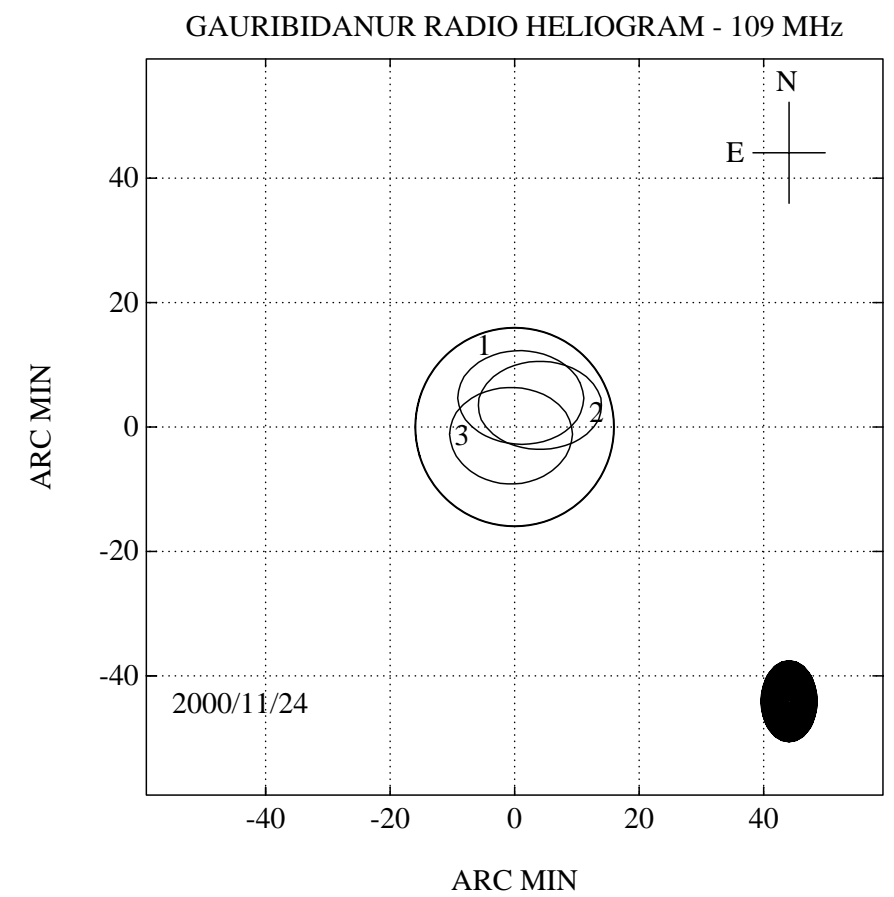

Fig. 5. Half-power contours of the radioheliogram obtained at 04:58:54 (1), 04:59:21 (2) and 04:59:47 UT (3), the various stages of the observed quasi-periodic radio emission. There is a close correspondence between the location of the discrete regions of intense emission in the radio image and the flare site mentioned in the text. The estimated peak radio brightness temperature is $\approx 2.92 \times 10^{8} \mathrm{~K}$ and it corresponds to the image obtained at 04:59:47 UT. The open circle at the center is the solar limb. The size of the GRH beam at $109 \mathrm{MHz}$ is indicated at the lower right corner.

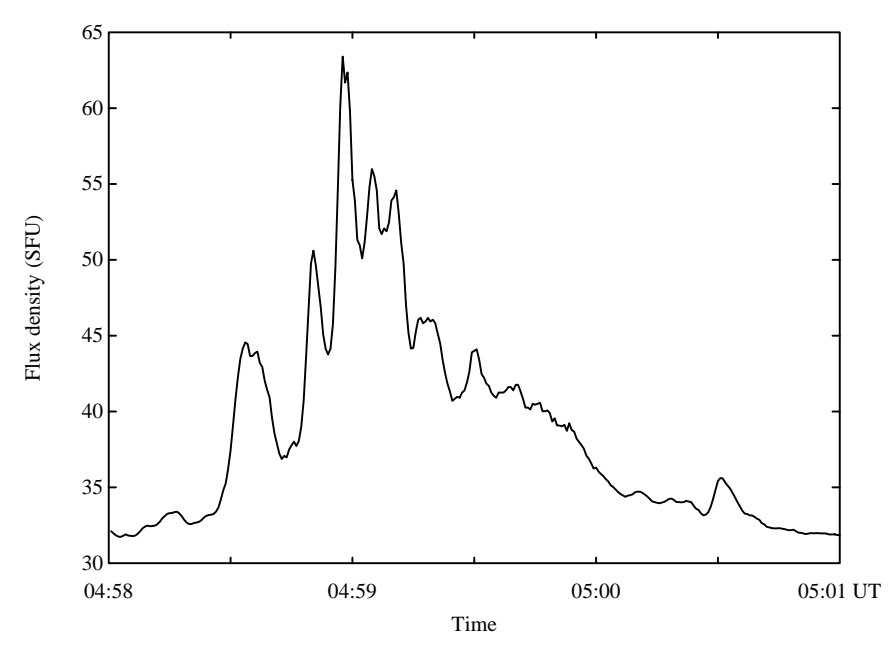

Fig. 6. Whole Sun flux observed with the GRH on November 24, 2000 during the period 04:58-05:01 UT. One can notice clear quasiperiodicity in the emission from 04:58:30 to 04:59:30 UT.

One of the suggested reasons for the above behaviour is the modulation of the electron beam injection/acceleration process responsible for the type III emission by quasi-periodic oscillations setup by propagating MHD waves (Aschwanden et al. 1994). We would like to note here that Klassen et al. (1999) 
had recently reported that groups of fast drift bursts or pulsations with a restricted bandwidth are observed in coronal loops during the impulsive flare phase prior to the onset of type II emission. They addressed them as tracers of a propagating disturbance which later becomes the exciter of type II emission. According to them, the disturbance must be a MHD wave or plasma propelled in the ambient corona. These indicate that the quasi-periodicity in the observed type III emission in the present case could also be due to the passage of a MHD disturbance through the solar corona. The observations of a metric type II radio burst following the type III emission strengthens the above argument. Associating the speed of the disturbance to the coronal Alfvén speed $\left(v_{\mathrm{A}}\right)$, we calculated the latter using the following relation (Roberts et al. 1984),

$p=2.6 \frac{a}{v_{\mathrm{A}}}$

where $p$ is the pulsation period, and $a$ is the dimension of the electron injection/acceleration region. Based on the results of an earlier statistical study of type III radio burst groups by Aschwanden et al. (1994), we assumed $a=3500 \mathrm{~km}$ in the present case. Substituting for the different values in Eq. (1), we get $v_{\mathrm{A}} \approx 1034 \mathrm{~km} \mathrm{~s}^{-1}$. It is interesting to note that this is almost equal to the speed of the "halo" CME mentioned earlier. Correlating this with the temporal relationship between the type III and the "EIT wave" event in the present case, we postulate that both of them could have been driven by the same "halo" CME. It is to be noted here that the evidence for CME as the probable cause for the "EIT waves" has already been pointed out in the literature (Thompson et al. 1998). We would like to add here that Klassen et al. (2000) had found that a majority of the "EIT wave" events always start in the time window around the associated type III bursts. The magnetic field ( $B$, Gauss) at the location of the plasma level corresponding to the observed radio emission was calculated from the definition of Alfvén speed (assuming 10\% He abundance in the corona), i.e.,

$v_{\mathrm{A}}=2 \times 10^{11} B N_{\mathrm{e}}^{-1 / 2} \mathrm{~cm} \mathrm{~s}^{-1}$

where $N_{\mathrm{e}}\left(\mathrm{cm}^{-3}\right)$ is the electron density. According to Zhao et al. (1991), type III groups which have multiple components of relatively large dimensions as well as wider distribution of periodicities could be due to emission in the fundamental mode. Taking clue from the above, we assumed that the observed radio radiation in the present case is due to fundamental emission and substituted $N_{\mathrm{e}}=1.47 \times 10^{8} \mathrm{~cm}^{-3}$ (the plasma density corresponding to $109 \mathrm{MHz}$ ). The estimated value of $B$ from Eq. (2) is $\approx 6.3 \mathrm{G}$. On the other hand, if one were to assume that the observed type III group is due to harmonic emission, then $N_{\mathrm{e}}=3.67 \times 10^{7} \mathrm{~cm}^{-3}$ (the plasma density corresponding to $54.5 \mathrm{MHz}$ ). Upon substituting this in Eq. (2), we get $B \approx 3.1 \mathrm{G}$. We also made an estimate of the height of the source region using the theoretical relation,

$\Delta t=\frac{h}{v_{\mathrm{A}}}$

and the value is $\approx 0.23 R_{\odot}$. Here $\Delta t$ is the time difference between the lift-off of the "halo" CME and the onset of the observed quasi-periodic emision. We verified the above height estimate in an independent manner using the result obtained earlier by Roberts et al. (1984) on the relationship between the ratio of the source size to its location and the duration time of the quasi-periodic phase (see Fig. 8 in the above reference), and the value is $\approx 0.20 R_{\odot}$. The different estimates obtained by substituting for the various parameters in the above equations are listed in Table 1. Note that the plasma parameters derived here should be treated with some caution since the solar corona is highly inhomogeneous.

\section{Conclusions}

We estimated the plasma parameters in the solar corona in an independent manner assuming that the quasi-periodic radio emission observed with the GRH on November 24, 2000 is due to the passage of a MHD disturbance through the solar atmosphere. The estimated coronal Alfvén speed agrees well with the reported speed of the "halo" CME observed on that day around the same time as the radio event. The close association between the "halo" CME, quasi-periodicity in the type III emission, "EIT wave", coronal and interplanetary type II radio burst seem to indicate that all of them probably have their root cause in the "halo" CME, as pointed out by Cliver et al. (1999). The total duration of the observed radio emission, the number of individual pulses $(\approx 7$ in the present case) and their periodicity are in the range of the corresponding values reported in the statistical study of type III groups by Aschwanden et al. (1994). In addition to the above, the period of the observed temporal fluctuations in the radio emission is consistent with the characteristic time scale of MHD oscillations set up by a travelling disturbance through the corona (Aschwanden et al. 1999). The derived height of the $109 \mathrm{MHz}$ source region is consistent with the location of the corresponding plasma density level above an active region in the corona, obtained recently by Aschwanden \& Acton (2001) using YOHKOH data. The estimated magnetic field is higher by about a factor of $5(\approx 3$ for emission in the harmonic mode) compared to that derived using the empirical relation given by Dulk \& McLean (1978) corresponding to the height of the $109 \mathrm{MHz}$ emission in the present case. It is suggested that further observations of this type might be an useful tool for determining parameters of the coronal plasma, since radio data can be obtained with a high time resolution. Also the present work gives a possible tool for obtaining a handle on the conditions of "EIT wave" propagation in the solar atmosphere (Mann et al. 1999), and to understand the modulation of injection/acceleration of non-thermal electrons released during a flare (Asai et al. 2001). We would like to add here that similar observations with imaging radio instruments of higher spatial resolution like the Giant Metrewave Radio Telescope (GMRT, Swarup et al. 1991) might also be useful in addressing the issue of fragmented energy release during solar flares (Benz 1994; Aschwanden et al. 1994; Vlahos \& Raoult 1995).

Acknowledgements. The staff members of the Gauribidanur radio observatory are thanked for their help in data collection and maintenance of the antenna and receiver systems. We are grateful to B. J. Thompson and A. Warmuth for their help regarding the "EIT wave" event presented in this paper. The radio spectral data is due to the kind courtesy of M. Akioka, K. Hori and Y. Kubo. We thank them. S. Yashiro 


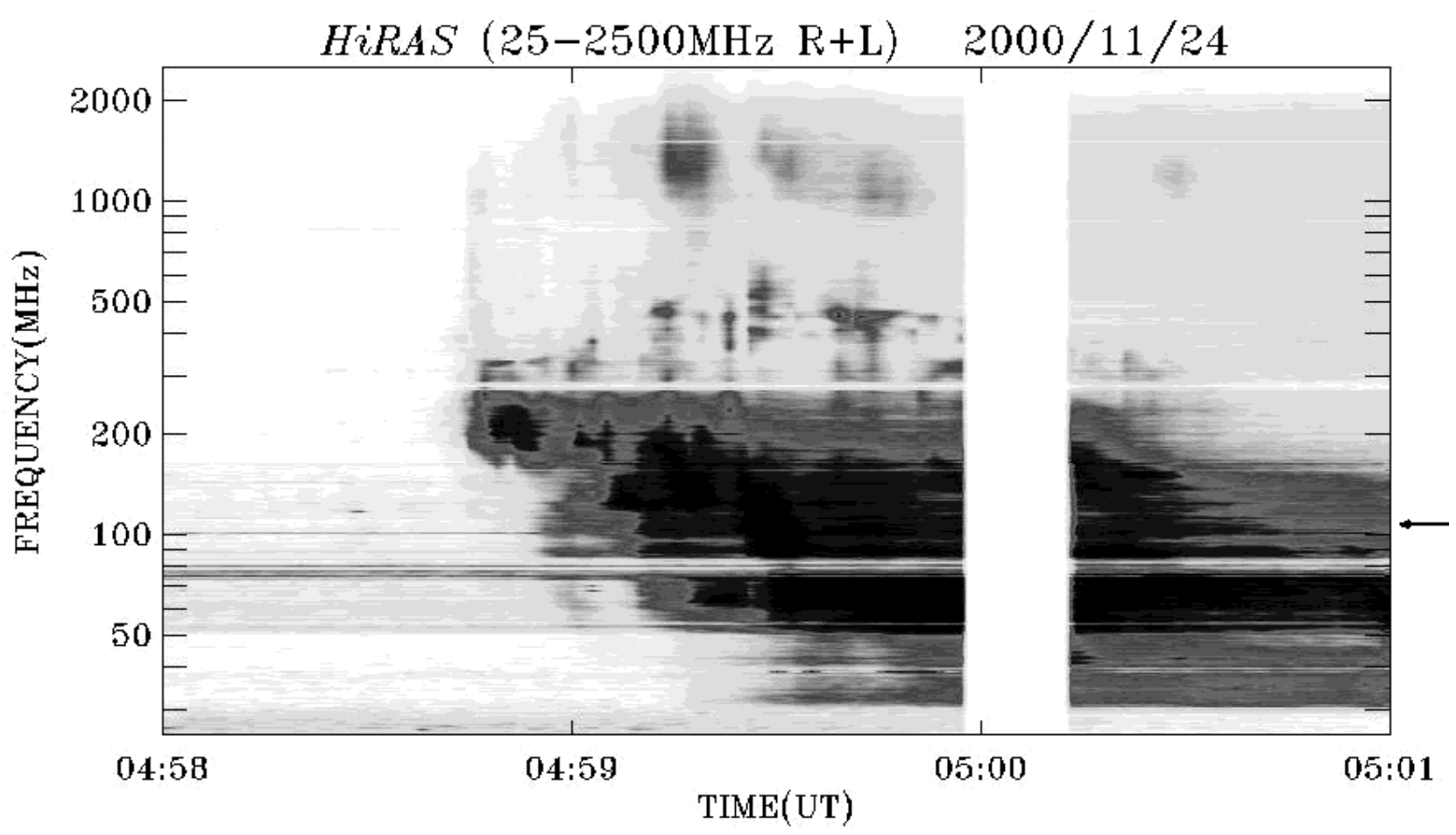

Fig. 7. Dynamic spectrum obtained by the Hiraiso radio spectrograph on November 24, 2000 during the period 04:58-05:01 UT. The arrow mark on the right margin indicates $f=109 \mathrm{MHz}$, the GRH observing frequency.

Table 1. Parameters related to the observed quasi-periodic emission.

\begin{tabular}{|c|c|c|c|c|c|c|c|c|}
\hline \multirow[t]{2}{*}{ Date } & \multirow{2}{*}{$\begin{array}{l}\text { CME } \\
\text { onset } \\
\text { (UT) }\end{array}$} & \multicolumn{3}{|c|}{ Quasi-periodic radio emission } & \multicolumn{2}{|c|}{ Magnetic field } & \multirow{2}{*}{$\begin{array}{l}\text { Alfvén } \\
\text { speed } \\
\left(\mathrm{km} \mathrm{s}^{-1}\right)\end{array}$} & \multirow{2}{*}{$\begin{array}{l}\text { Height of the } \\
\text { source region } \\
\qquad\left(R_{\odot}\right)\end{array}$} \\
\hline & & $\begin{array}{l}\text { onset } \\
\text { (UT) }\end{array}$ & $\begin{array}{l}\text { period } \\
(\mathrm{s})\end{array}$ & $\begin{array}{l}\text { duration } \\
\text { (s) }\end{array}$ & $\begin{array}{c}\text { Fundamental } \\
\text { (Gauss) }\end{array}$ & $\begin{array}{c}\text { Harmonic } \\
\text { (Gauss) }\end{array}$ & & \\
\hline $2000 / 11 / 24$ & $04: 55: 52$ & $04: 58: 30$ & 8.8 & 60 & 6.3 & 3.1 & 1034 & 0.23 \\
\hline
\end{tabular}

is thanked for providing details on the "halo" CME event reported. The SOHO data are produced by a consortium of the Naval Research Laboratory (USA), Max-Planck-Institut fuer Aeronomie (Germany), Laboratoire d'Astronomie (France), and University of Birmingham (UK). SOHO is a project of international cooperation between ESA and NASA. The CME catalog is generated and maintained by the Center for Solar Physics and Space Weather, the Catholic University of America in cooperation with the Naval Research Laboratory and NASA. We sincerely thank the referee whose critical comments on an earlier verison of this paper helped us in bringing out the results in a more clear fashion.

\section{References}

Asai, A., Shimojo, M., Isobe, H., et al. 2001, ApJ, 562, L103

Aschwanden, M. J. 1987, Sol. Phys., 111, 113

Aschwanden, M. J., Benz, A., \& Montello, M. L. 1994, ApJ, 431, 432

Aschwanden, M. J., Fletcher, L., Schrijver, C. J., \& Alexander, D. 1999, ApJ, 520, 880
Aschwanden, M. J., \& Acton, L. W. 2001, ApJ, 550, 475

Benz, A. O. 1994, Space Sci. Rev., 68, 135

Biesecker, D. A., Myers, D. C., Thompson, B. J., Hammer, D. M., \& Vourlidas, A. 2002, ApJ, 569, 1009

Brueckner, G. E., Howard, R. A., Koomen, M. J., et al. 1995, Sol. Phys., 162, 357

Cliver, E. W., Webb, D. F., \& Howard, R. A. 1999, Sol. Phys., 187, 89 Delaboudinière, J.-P., Artzner, G. E., Brunaud, J., et al. 1995, Sol. Phys., 162, 291

Dulk, G. A., \& McLean, D. J. 1978, Sol. Phys., 57, 279

Janssens, T. J., White, K. P., \& Broussad, R. M. III 1973, Sol. Phys., 31,207

Khan, J. I., \& Hudson, H. S. 2000, Geophys. Res. Lett., 27, 1083

Khan, J. I., \& Aurass, H. 2002, A\&A, 383, 1018

Klassen, A., Aurass, H., Klein, K.-L., Hofmann, A., \& Mann, G. 1999, A\&A, 343, 287

Klassen, A., Aurass, H., Mann, G., \& Thompson, B. J. 2000, A\&AS, 141,357

Kondo, T., Isobe, T., Igi, S., Watari, S., \& Tokumaru, M. 1995, J. Commun. Res. Lab., 42, 111

Mangeney, A., \& Pick, M. 1989, A\&A, 224, 242 
Mann, G., Aurass, H., Klassen, A., Estel, C., \& Thompson, B. J. 1999, in Plasma Dynamics and Diagnostics in the Solar Transition Region and Corona, ed. J.-C. Vial, \& B. Kaldeich-Schürmann, ESA SP-446, 477

Mercier, C. 1975, Sol. Phys., 45, 169

Pick, M., \& Ji, S. C. 1986, Sol. Phys., 107, 159

Pohjolainen, S., Maia, D., Pick, M., et al. 2001, ApJ, 556, 421

Ramesh, R., Subramanian, K. R., Sundararajan, M. S., \& Sastry, Ch. V. 1998, Sol. Phys., 181, 439

Roberts, B., Edwin, P. M., \& Benz, A. O. 1983, Nature, 305, 688

Roberts, B., Edwin, P. M., \& Benz, A. O. 1984, ApJ, 279, 857

Roberts, B. 2000, Sol. Phys., 193, 139

Roelof, E. C., \& Pick, M. 1979, A\&A, 210, 417

Solar Geophysical Data, 677, Part I, January 2001
Solar Geophysical Data, 681, Part II, May 2001

Swarup, G., Ananthakrishnan, S., Kapahi, V. K., et al. 1991, Current Sci., 60(2), 95

Thompson, B. J., Plunkett, S. P., Gurman, J. B., et al. 1998, Geophys. Res. Lett., 25(14), 2465

Thompson, B. J., Gurman, J. B., Neupert, W. M., et al. 1999, ApJ, $517, \mathrm{~L} 151$

Vlahos, L. 1986, in Energetic phenomenon on the Sun, ed. M. R. Kundu, \& B. Woodgate, NASA Conf. Publication, 2439, Chap. 2 Vlahos, L., \& Raoult, A. 1995, A\&A, 296, 844

Wills-Davey, M. J., \& Thompson, B. J. 1999, Sol. Phys., 190, 167

Wild, J. P., Smerd, S. F., \& Weiss, A. A. 1963, ARA\&A, 1, 291

Zhao, R.-y., Mangeney, A., \& Pick, M. 1991, A\&A, 241, 183 